\title{
LncRNA PCAT29 Suppresses Cell Proliferation, Invasion, and Mi- gration in Renal Carcinoma by Regulating FLOT1
}

\author{
Xiang Sun ${ }^{\text {a* }}$, Long-hua Luo ${ }^{\mathrm{a}}$, Ke-zhao Zhonga , Jia-rui Li ${ }^{\mathrm{a}}$ \\ ${ }^{a}$ Department of urology, the First Affiliated Hospital of Nanchang University, Nanchang, Jiangxi, 330006, China.
}

\begin{abstract}
Background: LncRNA PCAT29 has been reported to play a role in the development of cancer; the role of FLOT1 in renal carcinoma has been also identified. This study aimed at exploring the interaction between them and their influence on the progression of renal carcinoma.

Methods: The expression levels of lncRNA PCAT29 and the FLOT1 protein in tissues were determined separately using qRT-PCR and western blot, respectively. The endogenous expression of genes was modulated by recombinant plasmid and cell transfection. The cell viability, invasion, and migration were detected by MTT assay, transwell assay, and wound-healing cell migration assay, respectively. Any binding and interactions between the RNA and the proteins were determined with RNA immunoprecipitation and RNA pull-down assays. A nude mouse model for renal carcinoma was established for the in vivo expression of PCAT29.

Results: In renal carcinoma tissues, the expression of IncRNA PCAT29 was down-regulated while that of FLOT1 was up-regulated. PCAT29 negatively regulated FLOT1, and the overexpression of PCAT29 inhibited the cell proliferation viability, invasion, and migration of renal carcinoma by down-regulating FLOT1. The in vivo expression of PCAT29 inhibited tumor growth in a mouse model of renal carcinoma.

Conclusion: LncRNA PCAT29 inhibited the cell proliferation viability, invasion, and migration in renal carcinoma by down-regulating FLOT1, thereby suppressing tumor growth.

Keywords: LncRNA PCAT29; renal carcinoma; cell proliferation; cell invasion and migration; FLOT1
\end{abstract}

\section{INTRODUCTION}

Renal carcinoma, or renal cell carcinoma (RCC), is a common malignancy of the urinary system, surpassed in prevalence only by bladder neoplasm ${ }^{[1]}$. The incidence and mortality of RCC in different areas, genders, and ages are all with little optimism ${ }^{[2,3]}$. RCC originates from the renal proximal tubule, and $25-30 \%$ of patients with RCC have been found to have metastases; this diagnosis is grim as metastatic RCC is highly resistant to conventional chemotherapy ${ }^{[4]}$. The prognosis for metastatic RCC is poor because its therapeutic options are limited. Although radical nephrectomy can effectively cure early and local RCCs, 30\% of patients develop metastases after surgery ${ }^{[5,6]}$. Significant advances in the understanding of the tumor's biology and the molecular mechanisms underlying RCC metastasis

\footnotetext{
*Corresponding author: Xiang Sun, Ph.D.

Mailing address: Department of urology, the First Affiliated Hospital of Nanchang University, Nanchang. No. 17, Yongwaizheng Avenue, Donghu District, Nanchang, Jiangxi 330006, People's Republic of China.

TEL: +86-0791-88692526

E-mail: 121757633@qq.com
}

Received: 25 July 2018 Accepted: 25 September 2018 will contribute to the development of effective therapies and strategies for fighting metastasis.

The flotillin protein family has been proven to be a component of lipid rafts, in which flotillins were implicated in the initiation of signaling transduction via the recruitment of receptor kinases ${ }^{[7]}$. Flotillin-1 (FLOT1) is a member of the flotillin protein family and has been reported to be up-regulated in several kinds of cancers, such as hepatocellular carcinoma, breast cancer, and non-small cell lung cancer ${ }^{[8-10]}$. A high expression of FLOT1 is usually associated with cancer progression and a poor prognosis. It has been uniformly revealed that FLOT1 was up-regulated in human clear cell RCC, and it is also related to cancer progression and poor patient survival due to its enhancing effect on cell proliferation ${ }^{[11]}$. FLOT1 has been identified as a target gene of miR-506; it is negatively regulated by miR-506 and impacts the cell growth and metastasis incidence of RCC, exhibiting its crucial role in malignant cell behaviors ${ }^{[12]}$.

Long non-coding RNAs (lncRNAs) are RNA transcripts with more than 200 nucleotides and no protein coding potential that have recently been proved to be involved in the development and progression of human cancers $^{[13]}$. The prostate cancer-associated transcript 29 
(PCAT29) is a type of IncRNA and has been featured as an androgen-regulated tumor suppressor in prostate cancer ${ }^{[14]}$. PCAT29 has also been noted to be significantly dysregulated predominantly in one specific type of hepatitis virus-related hepatocellular carcinoma ${ }^{[15]}$. According to a previous study, the IncRNA DRAIC/ PCAT29 locus constitutes a tumor-suppressive nexus, and DRAIC expression predicts a good prognosis in clear cell RCC ${ }^{[16]}$. PCAT29, which is located $20 \mathrm{~kb}$ downstream of DRAIC, is also likely to have a positive effect on RCC progression, which deserves further exploration.

Whereas the significant role of FLOT1 in RCC ma has been verified, we speculated that PCAT29 may play a role in RCC by interacting with FLOT1. In this study, we investigated the interaction between PCAT29 and FLOT1 as well as their influence on cell proliferation viability, invasion, and migration in RCC, to clarify their roles in renal carcinoma development and progression and to offer insight to improve the prognosis for this type of tumor.

\section{MATERIALS AND METHODS}

Clinical samples

Twelve paired human RCC tissue and pericarcinomatous tissue samples were taken from patients who were admitted to the First Affiliated Hospital of Nanchang University and underwent surgery. The tissue samples were frozen in liquid nitrogen and stored at $-80^{\circ} \mathrm{C}$ before their pathological analysis. This research was approved by the Ethics Committee of the First Affiliated Hospital of Nanchang University and performed in accordance with the Declaration of Helsinki. All patients provided informed consent for tissue donation for this study before the samples were collected.

Total RNA extraction and quantitative real-time polymerase chain reaction

The total RNA was isolated from the tissues using a Trizol reagent (Invitrogen) according to the standard procedures of the manufacturer. The reverse transcription of the total RNA was performed using a cDNA Reverse Transcription Kit (Applied Biosystems) to synthesize the cDNA. The quantitative real-time polymerase chain reaction (qRT-PCR) was performed on an ABI 7900HT system (Applied Biosystems) with the SYBR Select Master Mix (Applied Biosystems). Primers were synthesized by Sangon Biotech (Shanghai, China). U6 and GAPDH served as internal control genes. The $2^{-\Delta \Delta C t}$ method was used to determine the relative expression level.

\section{Western blot}

Western blot was performed to analyze the protein expression level. The clinical tissues or cells were lysed in a lysis buffer (Beyotime Biotechnology) containing a protease inhibitor. The protein concentration was measured with a Bio-Rad protein assay system (Bio-Rad) and then separated by SDS-PAGE with an electrophoresis system (Bio-Rad). After separation, the proteins were transferred to a polyvinylidene difluoride (PVDF) membrane (Invitrogen). After blocking in Tris buffer saline (TBS) containing 5\% skim milk for $1 \mathrm{~h}$ at RT, the membrane was incubated with primary antibodies (Abcam, 1:1000) against FLOT1 or $\beta$-actin at $4^{\circ} \mathrm{C}$ for overnight. Then, the membrane was incubated with HRP-bounded antibodies for $2 \mathrm{~h}$ at RT. The target proteins were visualized using the ECL Plus Western Blotting Substrate (Thermo Scientific). $\beta$-actin was used as the control to quantify the protein level.

\section{Plasmid construction}

The pcDNA3.1 was used to construct the pcDNAPCAT29 and pcDNA-FLOT1 recombined plasmids. In brief, the target gene PCAT29 or FLOT1 was amplified by PCR, and the PCR products were purified with gel extraction. Then, the PCR products and pcDNA3.1 were integrated into pcDNA-PCAT2 9 and pcDNA-FLOT1 recombinant plasmids by T4 DNA ligase (Takara). The recombinant plasmids were cloned into E. coli, and the positive clones were amplified and selected. After restriction identification and sequence identification (Sangon Biotech), the recombinant plasmids were extracted from the positive clones and transfected into 786-0 cells for endogenous expression.

Cell culture and cell transfection

Human renal cancer cells 786-0 were cultured in DMEM supplemented with $10 \%$ fetal bosvine serum (FBS, Gibco), $100 \mathrm{U} / \mathrm{ml}$ of penicillin, and $100 \mu \mathrm{g} / \mathrm{ml}$ of streptomycin (Heclony) and maintained at $37^{\circ} \mathrm{C}$ in a humidified atmosphere with $5 \% \mathrm{CO}_{2}$.

The IncRNA PCAT29-specific siRNA (si-PCAT29), negative control (si-control), and si-FLOT1 were purchased from GenePharma Co., Ltd (Shanghai, China) for cell transfection. The 786-0 cells were seeded in 24-well plates and incubated overnight, then transiently transfected with si-PCAT29, si-control, si-FLOT1, and the recombined plasmids including pcDNA-PCAT29 and pcDNA-FLOT1 using Lipofectamine2000 (Invitrogen) according to the manufacturer's instructions.

\section{MTT assay}

Cell viability was determined by MTT assay. In total, $1 \times 10^{4}$ cells per well were seeded in 96-well plates and 
left overnight. Fresh media were used for the cells before they were incubated for another $48 \mathrm{~h}$. Then $20 \mu \mathrm{l}$ of MTT was added to each well and maintained for $4 \mathrm{~h}$ at $37^{\circ} \mathrm{C}$. After the media were removed and cultivation was terminated, the crystals were dissolved by adding $150 \mu \mathrm{l}$ DMSO and agitating the sample for $10 \mathrm{~min}$ at room temperature. An absorbance at $490 \mathrm{~nm}$ was measured on the microplate reader (Bio-Rad).

\section{Transwell assay}

A transwell chambers pre-coated Matrigel matrix (BD Bioscience) was used to conduct the invasion assay. The cells were cultured in serum-free media in the upper chamber of the transwell (Corning). A medium sump with $10 \%$ FBS was added to the lower chamber to function as a chemoattractant. After $12 \mathrm{~h}$ incubation at $37^{\circ} \mathrm{C}$, the cells that had invaded through the membrane were fixed with $4 \%$ formaldehyde (Sigma) for $15 \mathrm{~min}$ and stained in $0.1 \%$ crystal violet (Sigma). The stained cells were imaged and finally counted under a microscope (Olympus).

\section{Wound-healing cell migration assay}

The cells were cultured in six-well plates in complete media until they reached a confluent monolayer. Then, the complete media were replaced by serum-free media for cell culture. After being coated with gelatin, a wound was created by scraping a conventional pipette tip across the monolayer. A light microscope (Olympus) was used to determine the migration of cells toward the cell center.

\section{RNA pull-down assay}

An RNA pull-down assay was conducted to determine the interaction between PCAT29 and FLOT1. Briefly, the DNA probe complementary to PCAT29 was synthesized and biotinylated by GenePharma Co., Ltd. (Shanghai, China). An RNA pull-down assay was carried out using a Magnetic RNA-Protein Pull-Down Kit (Thermo Fisher) according to the manufactures' protocol. The RNA-binding protein complexes were washed and eluted for western blot or qRT-PCR analysis.

\section{RNA immunoprecipitation}

RNA immunoprecipitation (RIP) was performed with the RNA-Binding Protein Immunoprecipitation Kit (Millipore). The 786-0 cells were lysed with a lysis buffer, and the cell lysis solutions were incubated with AGO2 antibody or normal mouse IgG. The RNA-protein complexes were immunoprecipitated with protein $\mathrm{A}$ agarose beads, and the RNA was extracted using Trizol (Invitrogen). The IP-western was used to detect the AGO2 protein, and qRT-PCR was performed to quantify the PCAT29 and FLOT1.

Construction of a nude mouse model for renal carcinoma

All animal experiments were conducted in accordance with the National Institute of Health Guide for the Care and Use of Laboratory Animals and with the approval
A

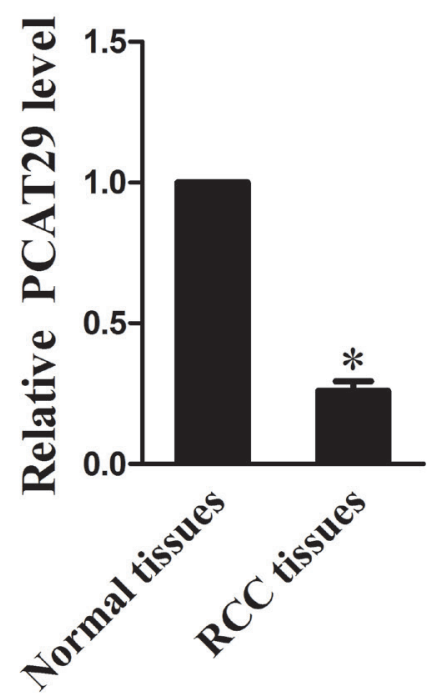

B

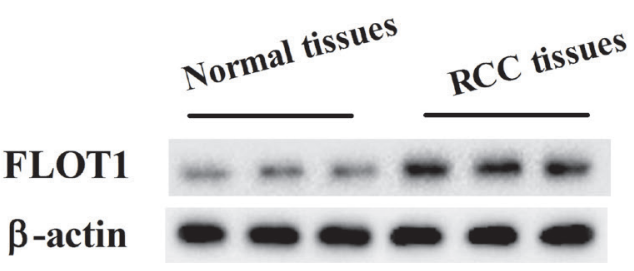

Figure 1. Altered expression of PCAT29 and FLOT1 in renal carcinoma tissues. The clinical renal carcinoma tissues (RCC, n=12) and pericarcinomatous normal tissues $(n=12)$ were obtained from patients with renal carcinoma. (A) The expression of PCAT29 in pericarcinomatous normal tissues and renal carcinoma tissues was quantified by qRT-PCR. (B) The FLOT1 protein level was determined with western blot. ${ }^{*} \mathrm{P}<0.05$ compared with normal tissues. 
A

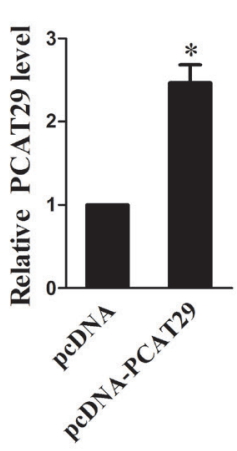

D

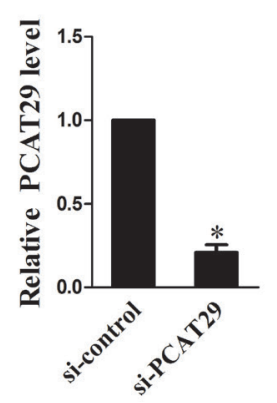

B

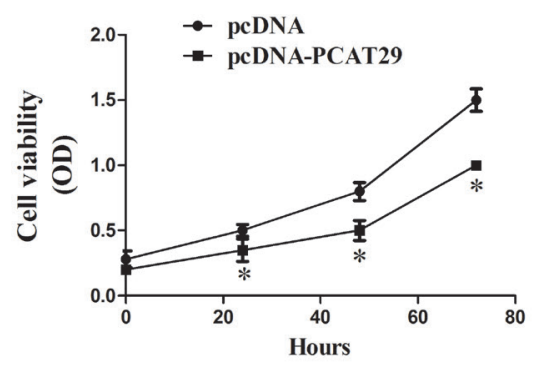

C
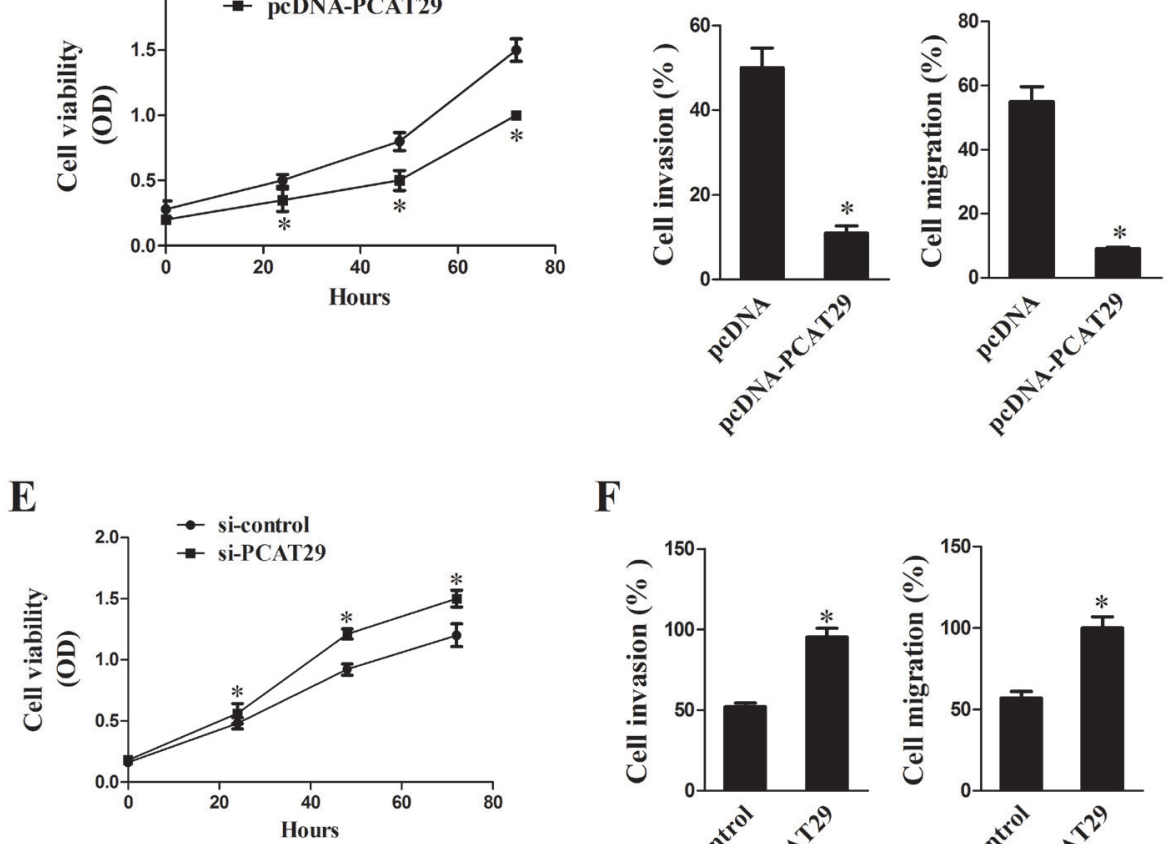

F

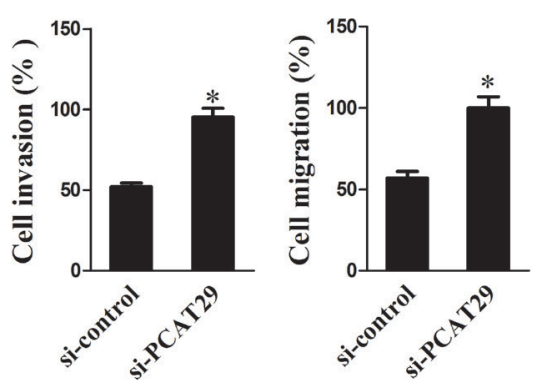

Figure 2. PCAT29 affected the cell proliferation viability, invasion, and migration of renal carcinoma. The $786-0$ renal carcinoma cells were transfected with pcDNA or pcDNA-PCAT29, and the expression of PCAT29 and its influence on cell growth were examined. (A) The expression of PCAT29 in 786-0 cells was quantified by qRT-PCR. (B) The viability of the 786-0 cells was determined by MTT assay. (C) Next, 786-0 cell invasion and migration were detected by transwell assay and wound-healing cell migration assay, respectively. ${ }^{*} \mathrm{P}<0.05$ compared with pcDNA. The 786-0 cells were transfected with pcDNA or pcDNA-PCAT29. (D) The expression of PCAT29 in 786-0 cells was quantified by qRT-PCR. (E) The viability of the 786-0 cells was determined by MTT assay. (F) The invasion and migration of the 786- 0 cells were detected by transwell assay and wound-healing cell migration assay, respectively. ${ }^{*} \mathrm{P}<0.05$ compared with the si-control.

of the Animal Care and Use Committee of the First Affiliated Hospital of Nanchang University. Ten health BALB/C female mice (aged 7 weeks) were purchased from the Cancer Research Center (Shanghai) and kept under specific pathogen-free conditions. Each mouse was injected with $1 \times 10^{8} 786-0$ cells transfected with pcDNA $(n=5)$ or pcDNA-PCAT29 $(n=5)$ in $0.2 \mathrm{ml}$ of PBS subcutaneously at the groin (25010867). All mice were sacrificed after 35 days to determine the tumor weight and gene expression.

\section{Statistical analysis}

Data were represented as the mean \pm standard deviation (SD) and were statistically analyzed using the Statistical Package for the Social Sciences (SPSS), version 21.0 (SPSS Inc.). Comparisons between the two groups were completed by Student's t-test, while comparisons among multi-groups were performed by one-way analysis of variance. A P-value $<0.05$ was considered statistically significant.

\section{RESULTS}

Altered expression of PCAT29 and FLOT1 in RCC tissues

Clinical renal carcinoma tissues $(n=12)$ and pericarcinomatous tissues $(n=12)$ were obtained from patients to determine their degree of expression of PCAT29 and FLOT1. Compared with the pericarcinomatous tissues $(n=12)$, the expression of PCAT29 was significantly decreased in RCC tissues ( $n=12)$ (Figure 1A), while the expression of FLOT1 was clearly increased in RCC tissues $(\mathrm{n}=12)$ (Figure 1B).

PCAT29 affected cell proliferation viability, invasion, and migration of RCC

Next, 786-0 RCC cells were transfected with pcDNA or pcDNA-PCAT29, and the expression of PCAT29 and its influence on cell growth were examined. It was revealed that the expression of PCAT29 was higher in cells transfected with pcDNA-PCAT29 compared with the pcDNA group (Figure 2A). Compared with pcDNA, the cell proliferation viability was repressed by pcDNA-PCAT29 (Figure 2B), and the cell invasion and 


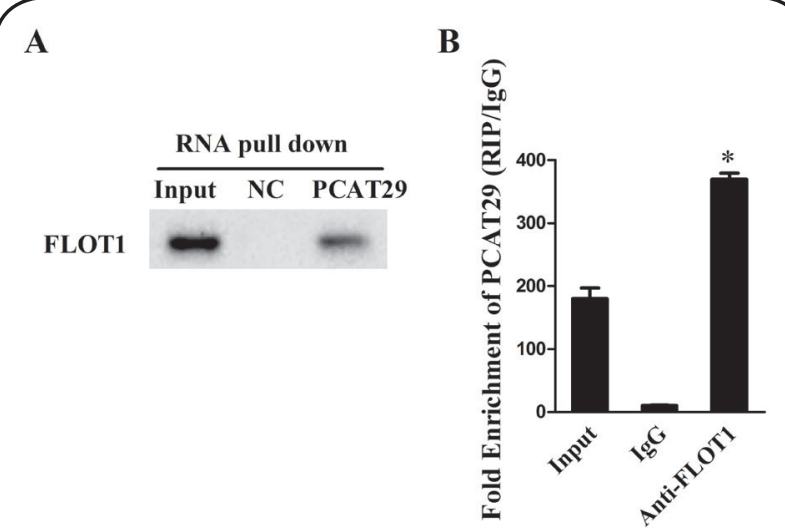

Figure 3. PCAT29 and FLOT1 interacted with each other in renal carcinoma cells. Binding and interaction between RNA and protein were determined with RNA immunoprecipitation (RIP) and RNA pull-down assay. (A) In the RNA pull-down assay, FLOT1 protein expression was determined using western blot. (B) In the RIP assay, the expression of PCAT29 in 786-0 cells was quantified with qRT-PCR. ${ }^{*} \mathrm{P}<0.05$ compared with IgG.

migration were also suppressed by pcDNA-PCAT29 (Figure 2C). The 786-0 cells were transfected with either the si-control or si-PCAT29, and then the expression of PCAT29 and its influence on cell growth were measured. Compared with the si-control, the expression of PCAT29 was lower in cells transfected with siPCAT29 (Figure 2D); the cell proliferation viability was also promoted by si-PCAT29 (Figure 2E). Finally, the cell invasion and migration were also facilitated by siPCAT29 (Figure 2F). These results suggested that when overexpressed, PCAT29 inhibited the cell proliferation viability, invasion, and migration in renal carcinoma.

PCAT29 and FLOT1 interacted with each other in RCC cells

In the RNA pull-down assay, FLOT1 in the pull-down complex of PCAT29 was analyzed by western blot. Compared with the NC (negative control), FLOT1 was detected in the pull-down complex of PCAT29 (Figure $3 \mathrm{~A})$, suggesting that an interaction existed between them. A RIP assay was used to analyze the binding between PCAT29 and FLOT1. Compared with IgG, PCAT29 accumulated extensively in the protein sample of FLOT1 (Figure 3B), suggesting binding between PCAT29 and FLOT1 had taken place.

\section{PCAT29 negatively regulated FLOT1}

By transfecting with different vectors, the 786-0 cells were divided into three groups: pcDNA, pcDNA-PCAT29, and pcDNA-PCAT29+pcDNA-FLOT1. The expression level of FLOT1 was also measured. Compared with pcDNA, pcDNA-PCAT29 suppressed the expression of FLOT1, which was reversed by pcD-

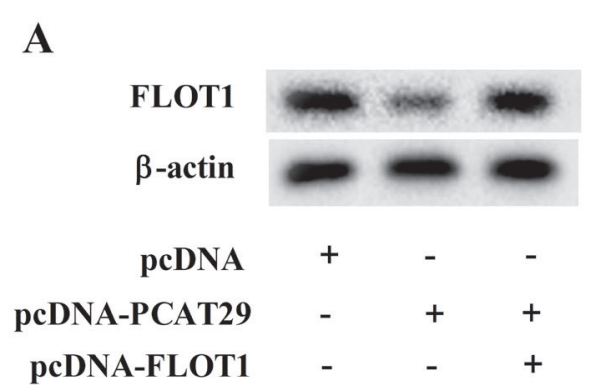

B

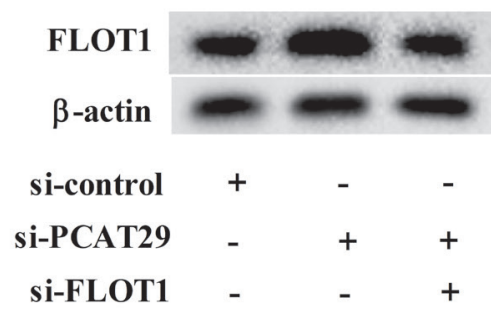

Figure 4. PCAT29 negatively regulated FLOT1. By transfecting them with different vectors, the 786-0 cells were divided into three groups: pcDNA, pcDNA-PCAT29, and pcDNAPCAT29+pcDNA-FLOT1. (A) The FLOT1 protein level was analyzed using western blot. Through cell transfection, the 7860 cells were divided into three groups: si-control, si-PCAT29, and si-PCAT29+si-FLOT1. (B) The expression level of FLOT1 was detected with a western blot.

NA-FLOT1 (Figure 4A). Through cell transfection, the 786-0 cells were divided into three groups: si-control, si-PCAT29, and si-PCAT29+si-FLOT1. The expression level of FLOT1 was also detected. Compared with the si-control, si-PCAT29 enhanced the expression of FLOT1, which was reversed by si-FLOT1 (Figure 4B).

When overexpressed, PCAT29 inhibited the cell proliferation viability, invasion and migration of RCC by down-regulating FLOT1

With different vectors transfected, the 786-0 cells were assigned to three groups: pcDNA, pcDNA-PCAT29, and pcDNA-PCAT29+pcDNA-FLOT1. The cell growth condition was also evaluated. Compared with pcDNA, pcDNA-PCAT29 inhibited the cell proliferation viability, which was reversed by pcDNA-FLOT1 (Figure 5A); the cell invasion and migration were also repressed by pcDNA-PCAT29 but reversed by pcDNA-FLOT1 (Figure $5 B)$.

Endogenously expressed PCAT29 inhibited tumor growth in a mouse model with RCC

Tumor xenograft nude mouse models were constructed and injected with pcDNA ( $\mathrm{n}=5$ ) or pcDNA-PCAT29 $(n=5)$, and the tumor weight was assessed. It was revealed that the tumor weight was clearly lower in mice expressing pcDNA-PCAT2 9 than in those that 


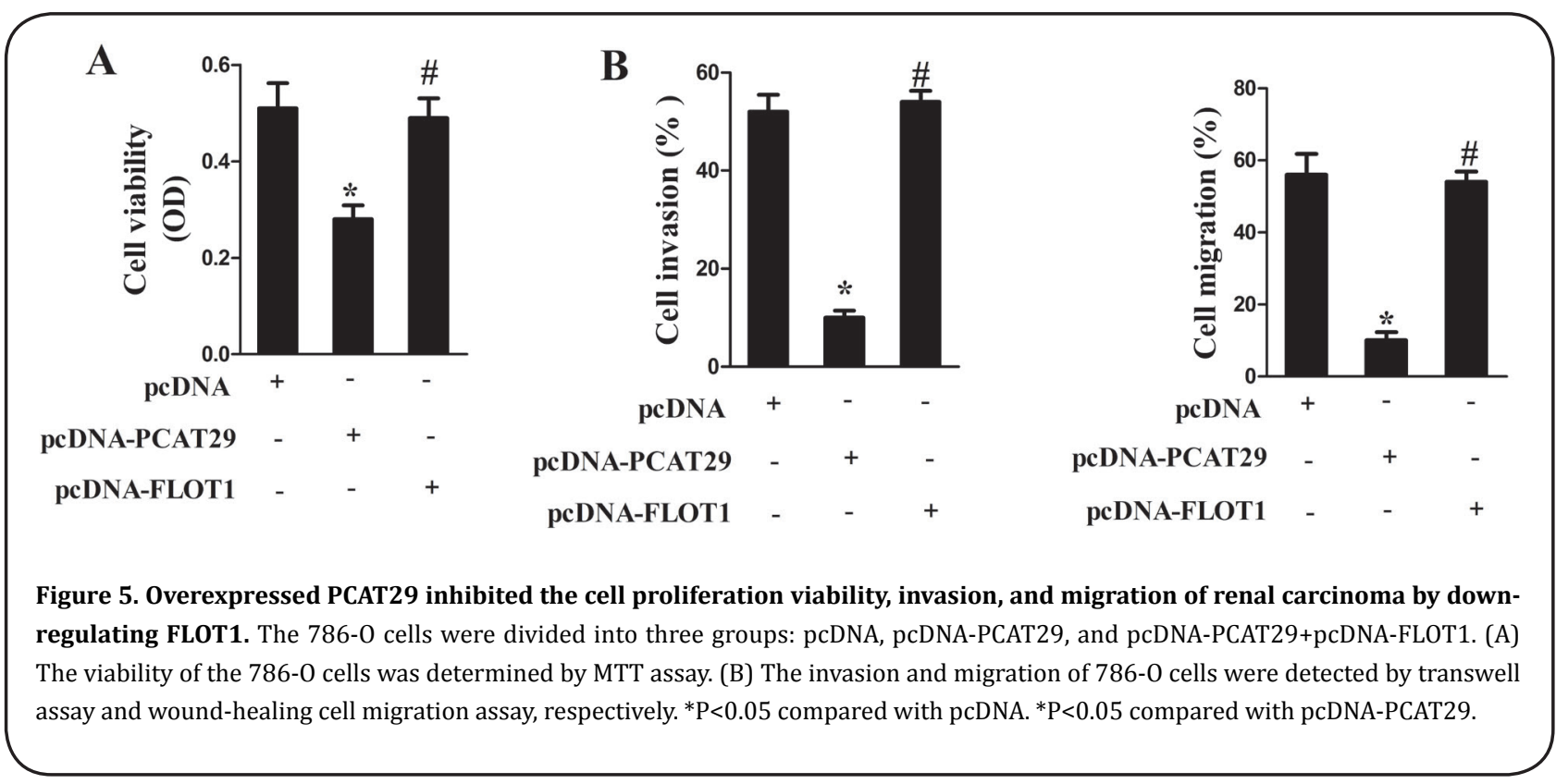

expressed pcDNA (Figure 6A); the expression of PCAT29 was also significantly higher in mice with pcDNA-PCAT29 (Figure 6B), while the expression of FLOT1 was distinctly lower in mice with pcDNA-PCAT 29 (Figure 6C). These results suggested that the in vivo expression of PCAT29 inhibited tumor growth in a mouse model of RCC.

\section{DISCUSSION}

In this study, the expression levels and interaction between IncRNA PCAT29 and FLOT1 in RCC were investigated, and their influence on the cell proliferation viability, invasion, and migration was also explored. LncRNA PCAT29 has been thought to have a low expression in RCC tissues and to inhibit the tumor cell proliferation viability, invasion, and migration by down-regulating FLOT1, which implicates its antitumor role in RCC development and progression. This study provides a significant perspective for developing novel therapies and improving the prognosis of renal carcinoma.

As an aggressive and lethal cancer as well as the second most common urologic cancer, RCC is known for its poor prognosis due to metastasis and its recurrent resistance to routine therapies ${ }^{[17]}$. In the past decade, notable progress has been realized in the treatment of RCC with the recent approval of new therapeutic agents, such as targeted therapy with VEGF and mTOR inhibition; the outcome has been significantly improved ${ }^{[18]}$. However, in advanced and metastatic RCC, treatment with conventional cytotoxic chemotherapy remains a challenge; metastatic RCC has been widely demonstrated to have a rare complete response to target drugs ${ }^{[4]}$. Tumor metastasis is a complex and multi-factorial process that involves complicated signal pathways in the tumor microenvironment; the activation and interactions of these pathways are closely implicated in the metastasis, invasion, survival, and growth of tumors ${ }^{[19]}$. Therefore, a better understanding of the pathogenesis and progression of RCC will require new therapies with improved outcomes.

After miRNAs, lncRNAs have recently been implicated in many human disorders for their regulator roles; the dysregulation of IncRNAs serves as a causal factor in tumorigenesis and the progression of cancers ${ }^{[20]}$. As emerging stars in gene regulation, epigenetics, and human disease, IncRNAs have attracted much attention from researchers searching for the pathogenesis and best treatments for cancer. In urologic cancers, such as prostate, bladder, and kidney cancer, lncRNAs usually function as epigenetic regulators of gene expression, competing endogenous RNA, and host genes for small noncoding RNAs deregulated in cancer ${ }^{[21]}$. MALAT1 is a common IncRNA and has been noted to be up-regulated in renal carcinoma and is correlated with tumor progression and a poor prognosis by interacting with Ezh2 and miR-205 ${ }^{[22,23]}$. LncRNA PCAT29 is an androgen-regulated tumor suppressor initially identified in prostate cancer and has been recently proven to be related to hepatitis virus-related hepatocellular carcinoma ${ }^{[15,24]}$. In this study, we determined that PCAT29 was down-regulated in renal carcinoma and inhibited tumor growth by suppressing cell proliferation viability, invasion, and migration, revealing its antitumor role in renal carcinoma. In addition, we discovered FLOT1 as a novel target of PCAT29, which is negatively regulated by PCAT29 and exerts an impact on cell prolifera- 


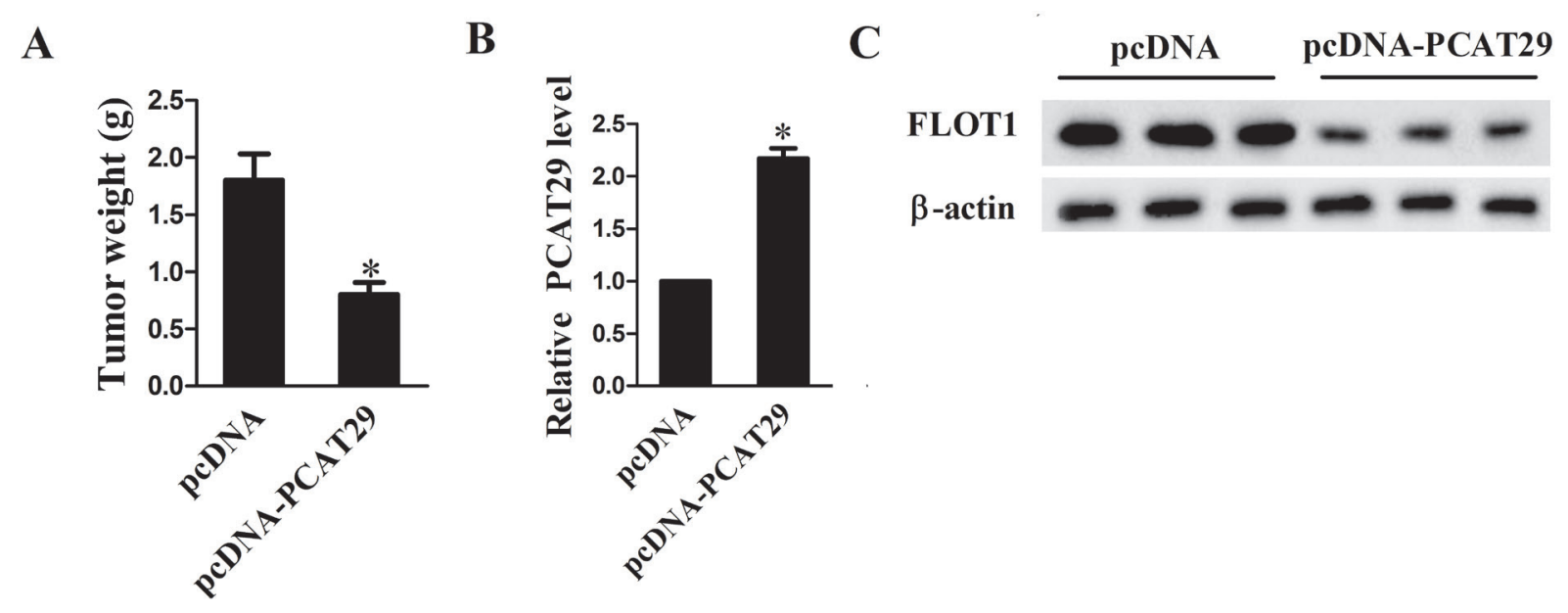

Figure 6. Endogenously expressed PCAT29 inhibited tumor growth in a mouse model of renal carcinoma. Tumor xenograft nude mouse models were constructed and injected with pcDNA $(n=5)$ or pcDNA-PCAT29 $(n=5)$. (A) The tumor weight of the mice in these two groups was assessed. (B) The expression of PCAT29 in renal carcinoma tissues was quantified using qRT-PCR. (B) The FLOT1 protein level was determined with a western blot. ${ }^{*} \mathrm{P}<0.05$ compared with pcDNA.

tion viability, invasion, and migration to repress tumor growth of renal carcinoma. It can be speculated that PCAT29 may suppress tumor metastasis by targeting FLOT1 in renal carcinoma, which may be of great benefit in improving the prognosis of renal carcinoma. This suspicion deserves further verification to be carried out in the future.

FLOT1 has been reported to be up-regulated in several types of cancers, and a high expression of FLOT1 is usually associated with progression and a poor prognosis for cancer ${ }^{[25]}$. FLOT1 has also been reported to be up-regulated in human clear cell RCC, and it is related to cancer progression and poor patient survival via enhancing cell proliferation ${ }^{[11]}$. In nasopharyngeal carcinoma, FLOT1 is up-regulated and promotes invasion and metastasis by activating TGF- $\beta$ signaling ${ }^{[26]}$. In our present study, we determined that FLOT1 was up-regulated in RCC, which led to cancer progression by facilitating the cell proliferation viability, invasion, and migration. Innovatively, we identified a regulatory relationship between PCAT29 and FLOT1, providing a potential therapeutic strategy for designing a molecular target for the treatment of RCC and other FLOT1-related cancers.

In this study, we investigated the interaction between PCAT29 and FLOT1 and their influence on the cell proliferation viability, invasion, and migration in RCC. We concluded that PCAT29 inhibited the cell proliferation viability, invasion and migration in RCC by down-regulating FLOT1 and thereby suppressing tumor growth. This study highlighted the roles of PCAT29 and FLOT1 in RCC development and progression and offered insights into the optimal management of its prognosis.

\section{REFERENCES}

1. Jonasch, E., Gao, J., and Rathmell, W. K. (2014) Renal cell carcinoma. BMJ 349, g4797

2. Znaor, A., Lortet-Tieulent, J., Laversanne, M., Jemal, A., and Bray, F. (2015) International variations and trends in renal cell carcinoma incidence and mortality. Eur Urol 67, 519-530

3. King, S. C., Pollack, L. A., Li, J., King, J. B., and Master, V. A. (2014) Continued increase in incidence of renal cell carcinoma, especially in young patients and high grade disease: United States 2001 to 2010. J Urol 191, 16651670

4. Mattei, J., da Silva, R. D., Sehrt, D., Molina, W. R., and Kim, F. J. (2014) Targeted therapy in metastatic renal carcinoma. Cancer Lett 343, 156-160

5. Jiang, Z., Chu, P. G., Woda, B. A., Liu, Q., Balaji, K. C., Rock, K. L., and Wu, C. L. (2008) Combination of quantitative IMP3 and tumor stage: a new system to predict metastasis for patients with localized renal cell carcinomas. Clin Cancer Res 14, 5579-5584

6. Shinohara, N., and Abe, T. (2015) Prognostic factors and risk classifications for patients with metastatic renal cell carcinoma. Int J Urol 22, 888-897

7. Riento, K., Frick, M., Schafer, I., and Nichols, B. J. (2009) Endocytosis of flotillin-1 and flotillin-2 is regulated by Fyn kinase. J Cell Sci 122, 912-918

8. Zhang, S. H., Wang, C. J., Shi, L., Li, X. H., Zhou, J., Song, L. B., and Liao, W. T. (2013) High Expression of FLOT1 Is Associated with Progression and Poor Prognosis in Hepatocellular Carcinoma. PLoS One 8, e64709

9. Li, H., Wang, R. M., Liu, S. G., Zhang, J. P., Luo, J. Y., Zhang, B. J., and Zhang, X. G. (2014) Abnormal expression of 
FLOT1 correlates with tumor progression and poor survival in patients with non-small cell lung cancer. Tumour Biol 35, 3311-3315

10. Lin, C., Wu, Z., Lin, X., Yu, C., Shi, T., Zeng, Y., Wang, X., Li, J., and Song, L. (2011) Knockdown of FLOT1 impairs cell proliferation and tumorigenicity in breast cancer through upregulation of FOXO3a. Clin Cancer Res 17, 3089-3099

11. Zhang, Y., Li, J., Song, Y., Chen, F., Pei, Y., and Yao, F. (2014) Flotillin-1 expression in human clear-cell renal cell carcinoma is associated with cancer progression and poor patient survival. Mol Med Rep 10, 860-866

12. Yang, F. Q., Zhang, H. M., Chen, S. J., Yan, Y., and Zheng, J. H. (2015) MiR-506 is down-regulated in clear cell renal cell carcinoma and inhibits cell growth and metastasis via targeting FLOT1. PLoS One 10, e0120258

13. Yang, G., Lu, X., and Yuan, L. (2014) LncRNA: a link between RNA and cancer. Biochim Biophys Acta 1839, 1097-1109

14. Malik, R., Patel, L., Prensner, J. R., Shi, Y., Iyer, M. K., Subramaniyan, S., Carley, A., Niknafs, Y. S., Sahu, A., Han, S., Ma, T., Liu, M., Asangani, I. A., Jing, X., Cao, X., Dhanasekaran, S. M., Robinson, D. R., Feng, F. Y., and Chinnaiyan, A. M. (2014) The IncRNA PCAT29 inhibits oncogenic phenotypes in prostate cancer. Mol Cancer Res 12, 10811087

15. Zhang, Q., Matsuura, K., Kleiner, D. E., Zamboni, F., Alter, H. J., and Farci, P. (2016) Analysis of long noncoding RNA expression in hepatocellular carcinoma of different viral etiology. J Transl Med 14, 328

16. Sakurai, K., Reon, B. J., Anaya, J., and Dutta, A. (2015) The IncRNA DRAIC/PCAT29 Locus Constitutes a Tumor-Suppressive Nexus. Mol Cancer Res 13, 828-838

17. Dabestani, S., Marconi, L., Hofmann, F., Stewart, F., Lam, T. B., Canfield, S. E., Staehler, M., Powles, T., Ljungberg, B., and Bex, A. (2014) Local treatments for metastases of renal cell carcinoma: a systematic review. Lancet Oncol
15, e549-561

18. Parekh, H., and Rini, B. I. (2015) Emerging therapeutic approaches in renal cell carcinoma. Expert Rev Anticancer Ther 15, 1305-1314

19. (2014) [Metastasized renal cell carcinoma - research subjects needed!]. Aktuelle Urol 45, 99

20. Kazemzadeh, M., Safaralizadeh, R., and Orang, A. V. (2015) LncRNAs: emerging players in gene regulation and disease pathogenesis. J Genet 94, 771-784

21. Martens-Uzunova, E. S., Bottcher, R., Croce, C. M., Jenster, G., Visakorpi, T., and Calin, G. A. (2014) Long noncoding RNA in prostate, bladder, and kidney cancer. Eur Urol 65, 1140-1151

22. Zhang, H. M., Yang, F. Q., Chen, S. J., Che, J., and Zheng, J. H. (2015) Upregulation of long non-coding RNA MALAT1 correlates with tumor progression and poor prognosis in clear cell renal cell carcinoma. Tumour Biol 36, 29472955

23. Hirata, H., Hinoda, Y., Shahryari, V., Deng, G., Nakajima, K., Tabatabai, Z. L., Ishii, N., and Dahiya, R. (2015) Long Noncoding RNA MALAT1 Promotes Aggressive Renal Cell Carcinoma through Ezh2 and Interacts with miR205. Cancer Res 75, 1322-1331

24. Al Aameri, R. F. H., Sheth, S., Alanisi, E. M. A., Borse, V., Mukherjea, D., Rybak, L. P., and Ramkumar, V. (2017) Tonic suppression of PCAT29 by the IL- 6 signaling pathway in prostate cancer: Reversal by resveratrol. PLoS One 12, e0177198

25. Li, H., Zhang, Y., Chen, S. W., Li, F. J., Zhuang, S. M., Wang, L. P., Zhang, J., and Song, M. (2014) Prognostic significance of Flotillin1 expression in clinically N0 tongue squamous cell cancer. Int J Clin Exp Pathol 7, 996-1003

26. Cao, S., Cui, Y., Xiao, H., Mai, M., Wang, C., Xie, S., Yang, J., Wu, S., Li, J., Song, L., Guo, X., and Lin, C. (2016) Upregulation of flotillin-1 promotes invasion and metastasis by activating TGF-beta signaling in nasopharyngeal carcinoma. Oncotarget 7, 4252-4264 